\title{
REHABILITATION OF SEVERELY ATROPHIED UPPER JAW WITH INTRAOSSAL DENTAL IMPLANTS - CLINICAL CASE.
}

\author{
Cenchev Iv., Cholakova R., Cvetanov C., Mitarcheva Ir.* \\ Department of Oral Surgery, Faculty of Dental Medicin, \\ *Department of foreign languages, \\ Medical University - Plovdiv, Bulgaria
}

\begin{abstract}
:
Objective: The purpose of this presentation is to show the difficulty in prosthetization of a clinical case with a pronounced atrophy of the upper jaw and the various types and nature of restrictions imposed by the requirements of the patient.

Methods: The clinical analysis, surgical protocol and prosthetic solution are presented in the treatment of 72 yearold woman with a pronounced atrophy of the upper jaw.

OPG, standard CT of the upper jaw was used in the planning and a special surgical template was fabricated, helping us to find intraoperatively the exact locations of implants. The preliminary analysis of the number, height and diameter of intraossal implants helped us to find the exact prosthetic solution in this clinical case. The preparation of the implant bed was done by conical osteotomy in order to expand and condense the existing bone, which allowed us to use endoossal implants with a possible maximum size in a very limited maxillary volume and the reluctance of the patient to use other methods and surgical techniques. Conical threaded and self-tapping intraossal implants were used, placed according to a classic two-stage methodology with a flap and a long-term functional loading after a period of four months.

Results and Conclusion: The applied surgical and prosthetic solution allowed us to achieve a good functional and aesthetic rehabilitation in this case of severe atrophy of the upper jaw, following a number of restrictions imposed on us by the reluctance of the patient to use other surgical solutions. This shows that in the case of severe atrophy of the upper jaw, a good clinical result can be achieved. For this reason, the use of CT, a well-planned surgical template, sufficient preparation, the maximum use of available bone volume and the choice of a good prosthetic solution is very important.
\end{abstract}

Key words: dental implants,osteotome techniques

\section{INTRODUCTION:}

Placing denture implants of completely tootles upper jaw posts a real challenge, especially if there is severe atrophy of the alveolar ridge. The above mentioned atrophy is a major obstacle for conventional denture treatment, as well as for the process of placementing of dental implants. The recuperation of upper jaw with dental implants is highly predictable and reliable process, provided there is enough bone tissue remaining on the jaw. Most dentists report high rate of success when using the method discussed - anywhere between 84-92\%.(1) However, the strong atrophy of the maxilla makes achieving this rate difficult. This is so because there is more strongly expressed atrophy of the alveolar bone on the vestibular side, After tooth loss, the thin buccal cortex of the maxillary alveolus resorbs to a greater degree than the thicker palatal socket wall /centripetal atrophy/. Parallel to this process, in the distal side of the jaw there is an increase of pneumatization of the maxillary sinuses and the atrophy of the alveolar ridge. In addition, the presence of the nasal cavity, and the most frequent D3-D4 bone density on the Lekholm\&Zarb scale, are all factors that combine to hamper the process of placementing dental implants in the jaw. (2)

There are several methods for improving the conditions for placing dental implants when there is atrophy of the upper jaw. Some of them are as follows:

- bone grafting -onlyan \& inlyan

- GBR

- nasal floor and maxillary sinus lifting

- techniques of placing implants that evade anatomy structures

- implants attached to adjacent structures/os zygomaticus ,proc.pterygoideus/

- Distraction osteogenesis

- reconstructive surgery (fracture Le Fort I type)

- osteotome techniques-bone(ridge) splitting ,bone spreding, bone condensation

All the above mentioned methods have their advantages, as well as disadvantages, and when used, have various success rate.

\section{MATERIALS AND METHODS}

The clinical case presented here is one of treating 72 year old woman with highly distinct atrophy of the upper jaw - third or fourth degree on the Cawood\&Howell scale (5). The 
patient has been dissatisfied with the previously performed rehabilitation of the upper jaw with conventional denture and opted for better solution of her problem. After having undergone thorough medical exams, the patient was informed of all the available options for her to choose from. Having analyzed the available options, pros and cons of each method, and the financial cost of each method, the patient and our team decided to place four dental implants by using osteotome techniques. Later, we would place a device, which a removable denture should be fixed to.

After analyzing KT (fig. 1.), the best places and sizes of the implants were determined, keeping in mind the specifics of the osteotome techniques we were going to use. Also, we had to take plaster cast of the patient jaw, and by using it, we produced a mould which was used later by our team to determine the exact and precise location of the implants. (fig. 2.) This very mould of the patient's jaw, after sterilizing, was used during the operation to help our team be guided to the exact spot we wanted to place the implants into. Using such an aid was very helpful because it enabled our team to work most efficiently with the remaining bone tissue. The team followed two stage surgical protocol where full-thickness flap was formed. After marking the spots of drilling with round drill, we used conic osteotomes (WavelineKohler) (fig. 3.), to shape the wholes for the implants. It took great precision to drill and widen the places where the implants were to be placed. The diameter of the wholes was made $3.2 \mathrm{~mm}$, and $3.7 \mathrm{~mm}$ respectively, which would be enough to fit standard implants of $3.7 \mathrm{~mm}$, and $4.2 \mathrm{~mm}$ diameter. Four conic self-screwing implants were placed - two $3,75 \times 10 \mathrm{~mm}$, and two $4.2 \times 10 \mathrm{~mm}$. The team was pleased with results because very satisfactory state of initial stability was achieved. The flap was sutured. (fig. 4, 5). There were no complications during the recovery period. The stitches were taken out after a week period. After a recovery period of four mounts, the implants were exposed, and a spleen structure was produced and attached to those bone implants. The spleen structure was used in turn to attach to it a reduced denture. (fig. 6, 7,8). In the end, the patient was highly satisfied with the achieved functional and aesthetic level of the denture structure. The patient had been examined every three months, for a period of eighteen months in total after the operation took place. There were no further complaints or complications registered.

\section{RESULTS AND DISCUSSION:}

Choosing the method for increasing thealveolar ridge bone depends on the surgeon experience, his preferences, the anatomical region, the degree and type of atrophy, the available distance between the jaws, the type of denture to be used, the aesthetical requirements, the number and length of surgical stages, the cost, and, of course, the desired by the patient results.
In the above described clinical case, our team choosed to use the osteotome techniques to improve the conditions for placing dental implants into the available bone. Our choice was also influenced by the unwillingness of the patient to undergo other types of surgical procedures, and by the opportunities the osteotome techniques presents for achieving good clinical and denture results.

The osteotome techniques for placing cylindrical dental implants was first described by Hilt Tatum in 1970. Later, the technique was modified and made popular by Summers in 1994, after he developed cylindrical osteomes which enable the forming of implant bedding for the vast majority of existing implant systems. (2)

The principles of this nonablative implant bed preparation technique are lateral and apical bone relocation and condensation., and their hardening afterward. By the virtue of this fact, the existing bone can be modified so the best size implant can be used, and the solidified bone provides increased initial stability which is very helpful for upcoming osteointegration. $(3,4,5)$

There are better conditions for implementation of osteotome techniques when dealing with the upper jaw, rather than the lower one. The main requirements for using the technique are:

Bone density D3-D4, minimal alveolar ridge width of at least $3 \mathrm{~mm}$, sufficient alveolar ridge height, lack of alveolar concavity, and plenty of distance between the jaws. $(2,5)$

The advantages of the osteotome techniques are the small number of surgical procedures, the possibility of direct installment of the dental implants, the improved quality of existing bone tissue, the high initial stability, and the low cost of the method. $(7,8)$

The drawbacks of the technique are the serious reabsorption of alveolar ridge bone in case of poorly performed surgery, and occasional cases of post operative vertigo. (6)

The precise planning and strict following of the rules for implementing the osteotome techniques are essential for the successful implant treatment. The mould our team used as an aid enabled us to transfer data from CT to the available alveolar ridge bone. In addition, the mould helped us determine the precise location of the implants, guided by the available $\mathrm{CT}$, without having to make a new one.

\section{CONCLUSION:}

Detailed clinical and para-clinical testing of patients, good planning in advance of surgical and denture treatment, tailoring the procedure to the patient's desires and expectations, and precise following of the surgical and denture protocol are guarantee for success and predictability of dental implant treatment. 
Fig. 1.
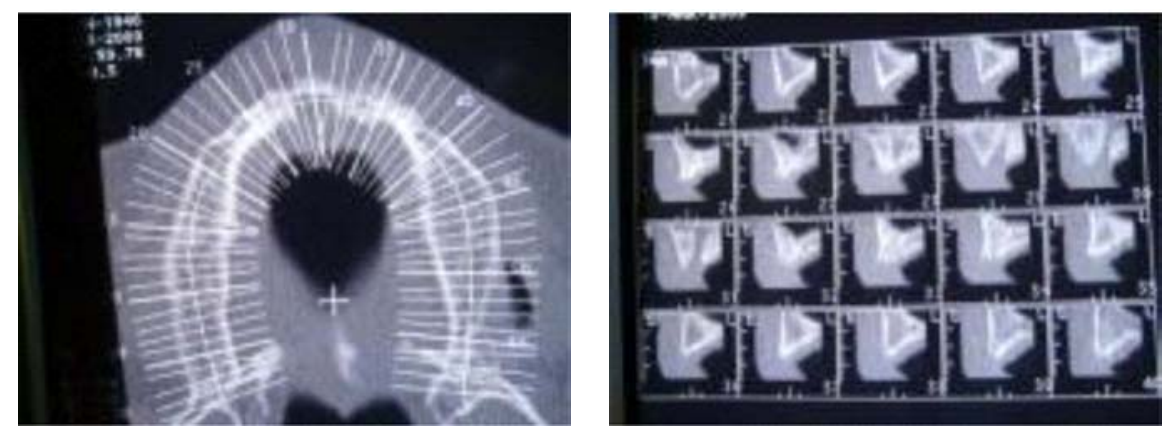

Fig. 2.
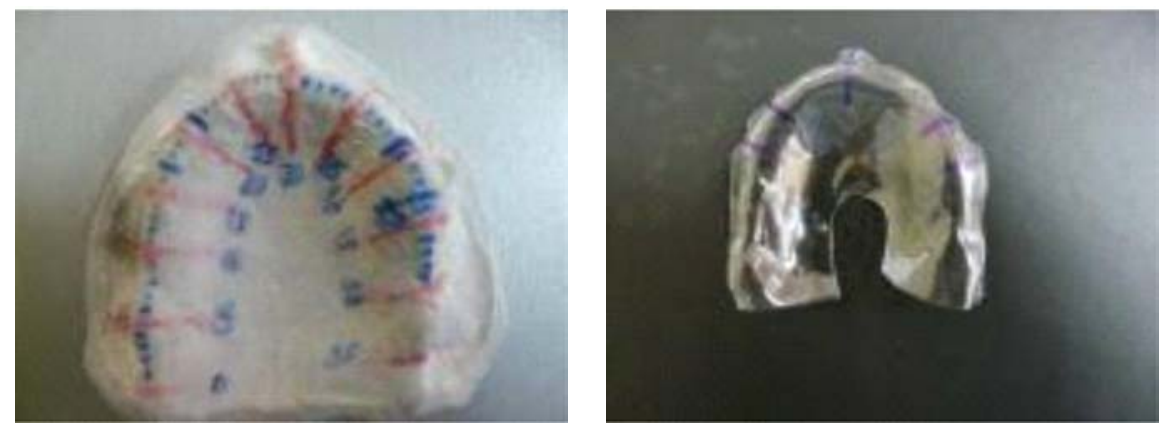

Fig. 3.
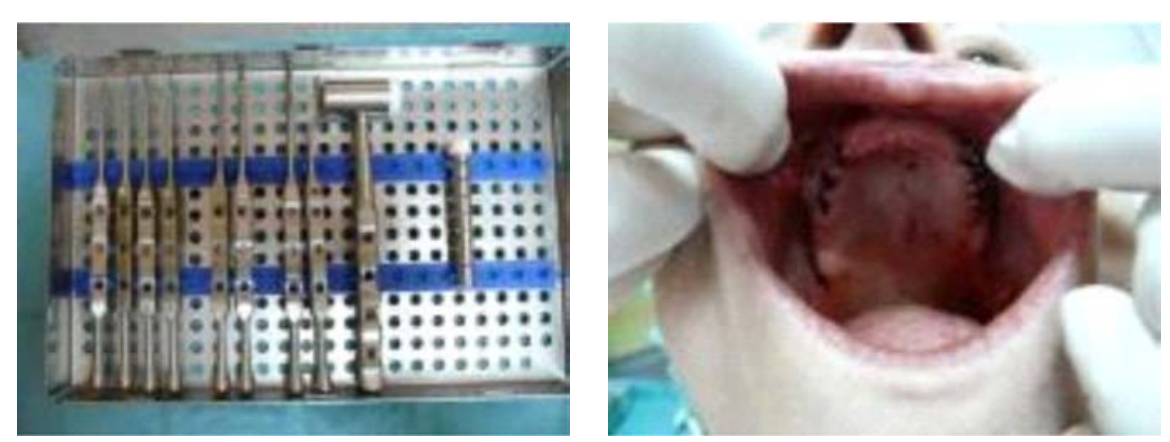

Fig. 4.

Fig. 5.
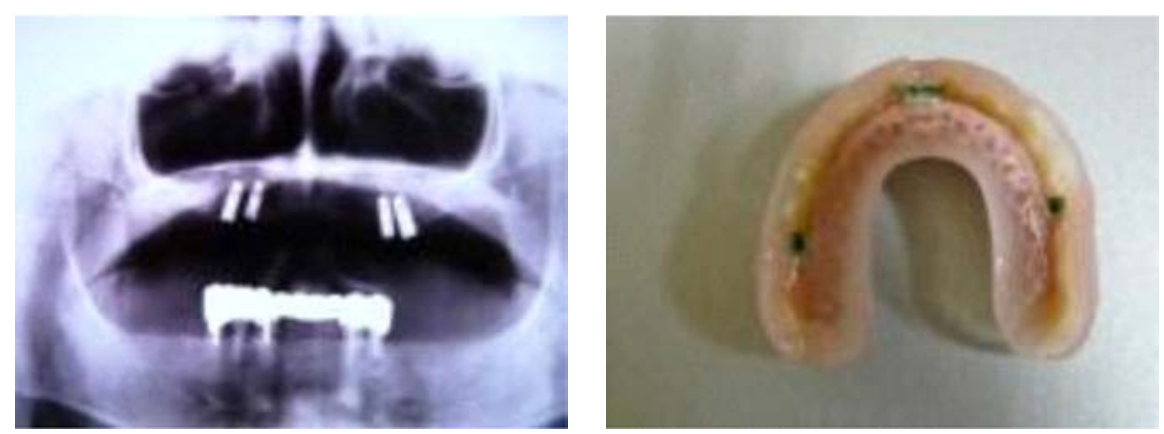

Fig. 6.
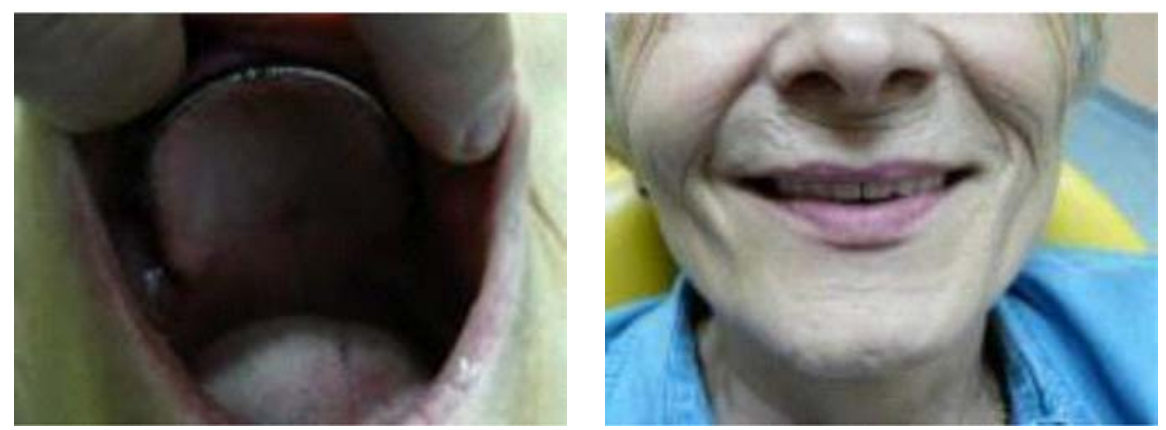

Fig. 8. 


\section{REFERENCES:}

1. Blanco J, Suarez J, Novio S, Villaverde G, Ramos I, Segade LA. Histomorphometric assessment in human cadavers of the peri-implant bone density in maxillary tuberosity following implant placement using osteotome and conventional techniques.

2. Carl E. Misch Contemporary Implant Dentistry, Third edition, Mosby Elsevier, 2008: 130-141, 178-197, 662-665.

3. Misch CM. Implant site development using ridge splitting techniques. Oral Maxillofac Surg Clin North Am. 2004 Feb; 16(1):65-74,
4. Penarrocha-Diago M, Rambla-Ferrer J, Perez V, Perez-Garrigues H, Benign paroxysmal vertigo secondary to placement of maxillary implants using the alveolar expansion technique with osteotomes: a study of 4 cases. Int $\mathrm{J}$ Oral Maxillofac Implants. 2008 Jan-Feb; 23(1):129-32.

5. Siddiqui AA, Sosovicka M. Lateral bone condensing and expansion for placement of endosseous dental implants: a new technique. J Oral Implantol. 2006; 32(2):87

6. Sorni M, Guarinos J, Garcia O, Penarrocha M. Implant rehabilitation of the atrophic upper jaw: a review of the literature since 1999. Med Oral Patol Oral Cir Bucal. 2005 Apr 1; 10 Suppl 1: E45-56.

7. Strietzel FP, Nowak M, Kuchler I, Friedmann A. Peri-implant alveolar bone loss with respect to bone quality after use of the osteotome technique: results of a retrospective study. Clin Oral Implants Res. 2002 Oct; 13(5):508-13.

8. Strietzel FP, Nowak M. Mund Kiefer Gesichtschir. Changes in the alveolar ridge level in implantation using the osteotomy technic. Retrospective studies. 1999 Nov; 3(6):309-13.

\section{Address for correspondence:}

D-r Ivan Chenchev

Department of Oral Surgery, Faculty of Stomatology, Medical University, Plovdiv 3,"Hristo Botev" bul., 4000 Plovdiv, Bulgaria

Mobile: $+359 / 88 / 8722321$

E-mail: ivan_chenchev@yahoo.com 